\title{
A NEW BOUNDARY ELEMENT TECHNIQUE FOR ONE- AND TWO- TEMPERATURE MODELS OF 3D BIOTHERMOMECHANICAL BEHAVIOR OF ANISOTROPIC BIOLOGICAL TISSUES
}

\author{
MOHAMED ABDELSABOUR FAHMY ${ }^{1,2}$ \\ ${ }^{1}$ Department of Basic Sciences, Faculty of Computers and Informatics, Suez Canal University \\ New Campus, 4.5 Km, Ring Road, El Salam District, 41522 Ismailia, Egypt. \\ E-mail: mohamed_fahmy@ci.suez.edu.eg \\ ${ }^{2}$ Department of Mathematics, Jamoum University College, Umm Al-Qura University \\ Alshohdaa 25371, Jamoum, Makkah, Saudi Arabia \\ E-mail: maselim@uqu.edu.sa and URL: https://uq.sa/wVmd0G
}

Key words: Dual Phase Lag Bioheat Transfer, Biothermomechanics, Anisotropic Biological Tissues, Boundary Element Method, Iterative Solver.

\begin{abstract}
The main objective of this paper is to develop a novel boundary element technique for describing the three-dimensional (3D) biothermomechanical behavior of anisotropic biological tissues. The governing equations are studied on the basis of the dual phase lag bioheat transfer and Biot's theory for one- and two-temperature models. Because of the benefits of CQBEM, such as not being restricted by the complex shape of biological tissues and not requiring discretization of the interior of the treated region, it can cope with complex bioheat models and has low use of RAM and CPU. CQBEM is therefore a flexible and efficient tool for modeling the distribution of bioheat in anisotropic biological tissues and associated deformation. The resulting linear equations arising from CQBEM are solved by the generalized modified shift-splitting (GMSS) iterative method which reduces the number of iterations and the total time of the CPU. Numerical findings show the validity, efficacy and consistency of the proposed technique.
\end{abstract}

\section{INTRODUCTION}

Human body is a complicated thermal structure, Arsene d'Arsonval and Claude Bernard have been proved that the temperature difference between arterial blood and venous blood is due to oxygenation of blood [1]. An important number of research papers in bioheat transfer over the past few decades has focused on an understanding blood flow effect on the temperature distribution within living biological tissues. The first attempt to describe the temperature distribution in biological tissues with blood flow effect has been introduced by Pennes [2]. Askarizadeh and Ahmadikia [3] solved analytically Fourier and non-Fourier bioheat equations in skin tissue. Li et al. [4] established the biothermomechanical behavior in bi-layered human skin.

Due to the nonlinearity of the bioheat equations, it is very difficult to solve them analytically $[5,6]$ in general, Therefore, many researchers have used and applied various numerical methods like finite difference method (FDM) [7-9], finite element method (FEM) [10] and boundary element method (BEM) [11-31]. The $\mathrm{BEM}$ is one of the numerical methods used to solve the current general problem [32-56]. Generally, Laplacedomain fundamental solutions are easier to obtain than time-domain fundamental solutions for engineering and 
scientific problems [57, 58]. Since the CQBEM requires Laplace-domain fundamental solutions of the problem's governing equations and didn't need unknown time-domain fundamental solutions. Therefore, it is widely used in scientific and engineering applications.

The main purpose of this article is to propose a novel boundary element model for describing thermomechanical interactions in anisotropic biological tissues. The uncoupled governing equations are solved independently, where the dual phase lag bioheat transfer equation is solved first for obtaining the temperature distribution using the boundary element method, and then the mechanical equation has been solved using the CQBEM to obtain the displacement components for different temperature distributions at each time step. The resulting linear equations arising from BEM are solved by GMSS which reduces the number of iterations and total CPU time.

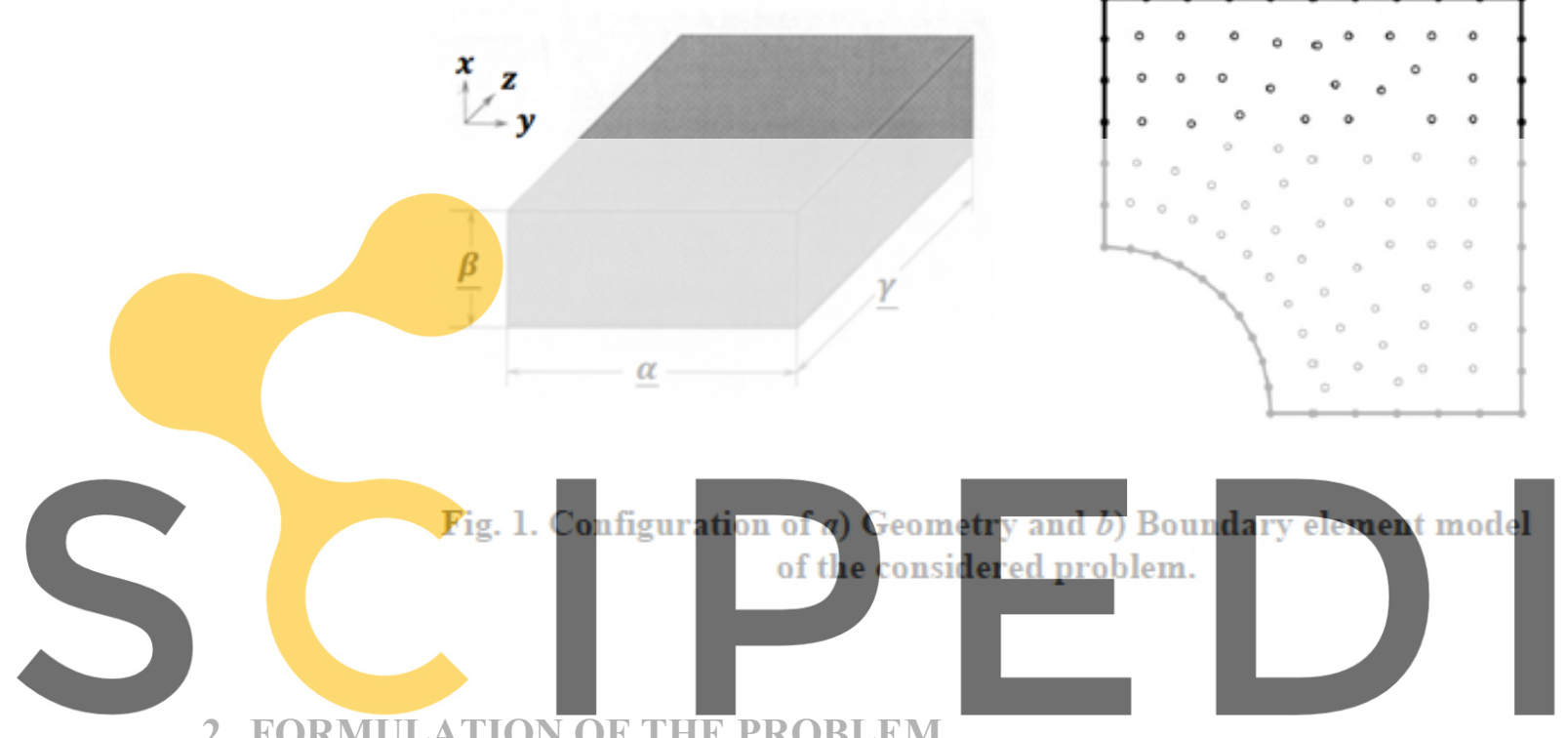

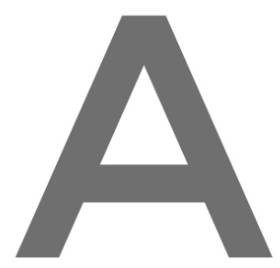

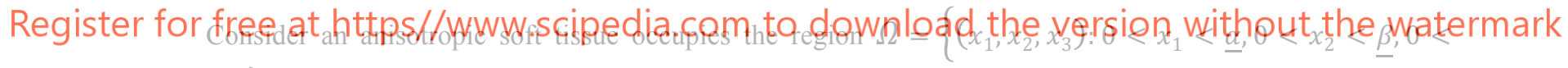

$\left.x_{3}<\underline{\gamma}\right\}$ with boundary $\Gamma$ as shown in Fig. $1 a$.

On the basis of Biot's theory $[59,60]$, the governing equations that model the biothermomechanical behavior of anisotropic biological tissues can be expressed as

$$
\begin{aligned}
& \left(\nabla^{T} \sigma\right)^{T}+F=\rho \ddot{u}+\phi \rho_{f}\left(\ddot{u}_{f}-\ddot{u}\right) \\
& \dot{\zeta}+\nabla^{T} q=0 \\
& \sigma=\left(C_{a j l g} \text { tr } \epsilon-A p\right) I-\mathfrak{B}\left(T-T_{0}\right) \\
& \epsilon=\frac{1}{2}\left(\nabla u^{T}+\left(\nabla u^{T}\right)^{T}\right) \\
& \zeta=\mathrm{A} \operatorname{tr} \epsilon+\frac{\phi^{2}}{\mathrm{R}} \mathrm{P}
\end{aligned}
$$

where the equation governing fluid motion has been modeled using the rule of Darcy [61]

$$
q=-K\left(\nabla p+\rho_{f} \ddot{u}+\frac{\rho_{a}+\phi \rho_{f}}{\phi}\left(\ddot{u}_{f}-\ddot{u}\right)\right)
$$

The 3D bioheat transfer equation expressed by the following dual phase lag model [62]:

$$
c \rho\left[\frac{\partial T}{\partial \tau}+\tau_{q} \frac{\partial^{2} T}{\partial \tau^{2}}\right]=\breve{K} \nabla^{2} T+\widetilde{K} \tau_{T} \frac{\partial}{\partial \tau}\left(\nabla^{2} T\right)+W_{b} C_{b}\left(\breve{T}_{b}-T\right)+Q_{m}+Q_{r}-W_{b} C_{b} \tau_{q} \frac{\partial T}{\partial \tau}(7)
$$


where the relation between the conductive temperature $T$ and the thermodynamic temperature $\phi$ is $\boldsymbol{T}-$ $\mu \boldsymbol{T}_{, x x}=\phi$ and $\mu$ is the two-temperature parameter which is also denoted the temperature discrepancy.

In the current study, we assumed that $\mathbb{C}=0.66$ at low frequency as measured by Bonnet and Auriault [63].

According to Bonnet [64], the equations of motion of our problem can be expressed as follows [65]

$$
\left.\begin{array}{ll}
\hat{B}_{\tilde{x}} \hat{u}^{g}(\tilde{x})=0 & \text { for } \tilde{x} \in \Omega \\
\hat{u}^{g}(x)=\hat{g}_{D} & \text { for } x \in \Gamma_{D} \\
\hat{t}^{g}(x)=\hat{g}_{N} & \text { for } x \in \Gamma_{N}
\end{array}\right\}
$$

where $\Gamma=\Gamma_{D} \cup \Gamma_{N}, \Gamma_{D} \cap \Gamma_{N}=\phi, \hat{B}_{\tilde{x}}$ and $\hat{t}^{g}$ are defined as

$$
\hat{B}_{\tilde{x}}=\left[\begin{array}{ccc}
B_{\tilde{x}}^{e}+s^{2}\left(\rho-\beta \rho_{f}\right) I & (\alpha-\beta) \nabla_{\tilde{x}} & -\mathfrak{B} \nabla_{\tilde{x}} \\
s(\alpha-\beta) \nabla_{\tilde{x}}^{T} & -\frac{\beta}{s \rho_{f}} \Delta_{\tilde{x}}+\frac{s \phi^{2}}{R} & 0
\end{array}\right], \hat{t}^{g}(x)=\left[\begin{array}{ccc}
T_{x}^{e} & -\alpha n_{x} & 0 \\
s \beta n_{x}^{T} & \frac{\beta}{s \rho_{f}} n_{x}^{T} \nabla_{x} & 0
\end{array}\right]\left[\begin{array}{l}
\hat{u}(x) \\
\hat{p}(x) \\
T(x)
\end{array}\right],
$$

In which $\beta=\frac{\phi^{2} s K \rho_{f}}{\phi^{2}+s K\left(\rho_{a}+\phi \rho_{f}\right)}$

\section{BOUNDARY ELEMENT IMPLEMENTATION FOR BIOHEAT TRANSFER}

\section{FIELD}

Through this section, our main goal is to outline a boundary element procedure for solving (7) subjected to the following initial and boundary conditions

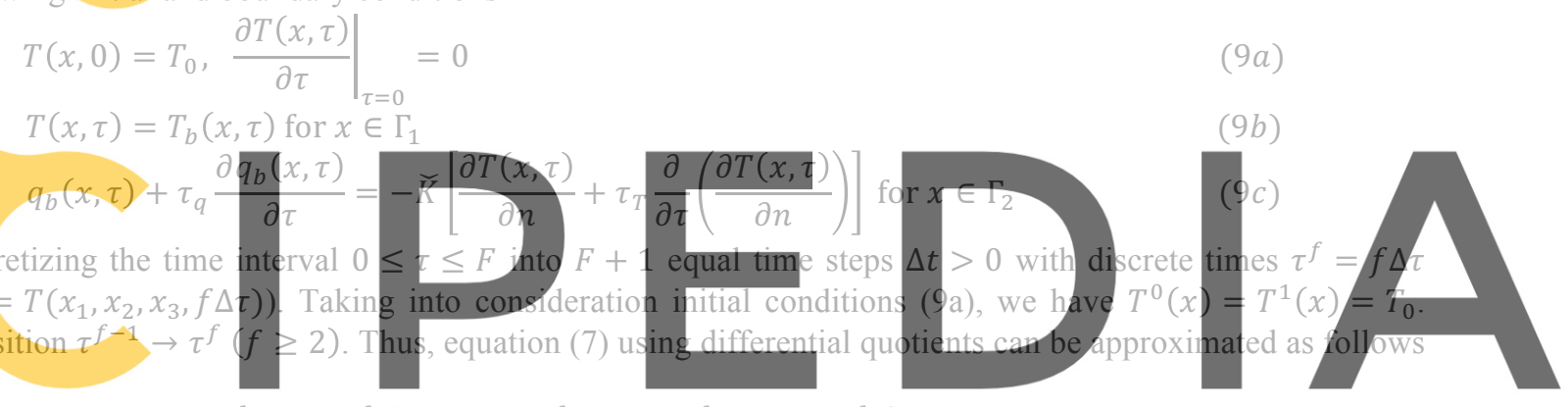

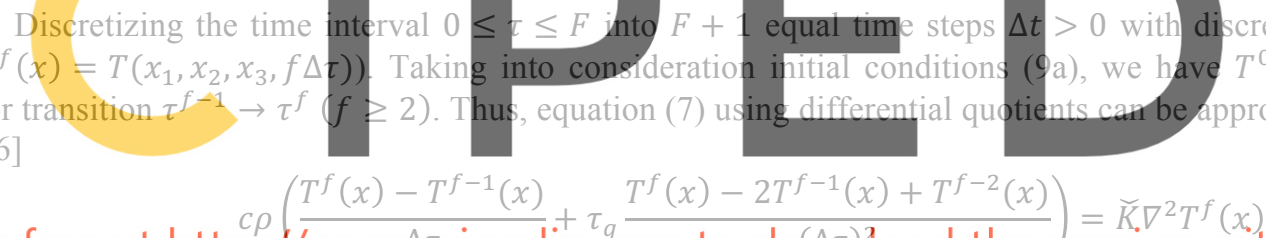

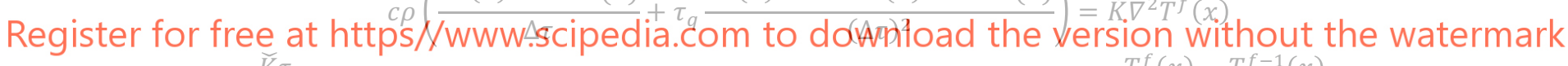

$$
+\frac{\breve{K} \tau_{T}}{\Delta \tau}\left[\nabla^{2} T^{f}(x)-\nabla^{2} T^{f-1}(x)\right]+W_{b} C_{b}\left[\breve{T}_{b}-T^{f}(x)\right]+Q_{m}+Q_{r}-W_{b} C_{b} \tau_{q} \frac{T^{f}(x)-T^{f-1}(x)}{\Delta \tau}(10)
$$

which can be written as follows

$$
\nabla^{2} T^{f}(x)-B T^{f}(x)+C \nabla^{2} T^{f-1}(x)+D T^{f-1}+E T^{f-2}(x)+F=0
$$

$$
\begin{aligned}
& B=\frac{\left(c \rho+W_{b} C_{b} \Delta \tau\right)\left(\Delta \tau+\tau_{q}\right)}{\widetilde{K} \Delta \tau\left(\Delta \tau+\tau_{T}\right)}, C=\frac{\tau_{T}}{\Delta \tau+\tau_{T}}, D=\frac{c \rho\left(\Delta \tau+2 \tau_{q}\right)+W_{b} C_{b} \tau_{q} \Delta \tau}{\widetilde{K} \Delta \tau\left(\Delta \tau+\tau_{T}\right)} \\
& E=-\frac{c \rho \tau_{q}}{\widetilde{K} \Delta \tau\left(\Delta \tau+\tau_{T}\right)}, F=\frac{\Delta \tau\left(W_{b} C_{b} \breve{T}_{b}+Q_{m}+Q_{r}\right)}{\widetilde{K}\left(\Delta \tau+\tau_{T}\right)} .
\end{aligned}
$$

The boundary conditions (9b) and (9c) can be reexpressed as

$$
\begin{aligned}
T^{f}(x) & =T_{b}^{f}(x) \text { for } x \in \Gamma_{1} \\
Z^{f}(x) & =-\breve{K} \frac{\partial T^{f}(x)}{\partial n}=w_{b}^{f}(x) \text { for } x \in \Gamma_{2}
\end{aligned}
$$

where

$$
w_{b}^{f}(x)=\frac{\Delta \tau}{\Delta \tau+\tau_{T}}\left(q_{b}^{f}(x)+\left.\tau_{q} \frac{\partial q_{b}(x, \tau)}{\partial \tau}\right|_{t=t^{f}}\right)-\widetilde{K} \frac{\tau_{T}}{\Delta \tau+\tau_{T}} \frac{\partial T^{f-1}(x)}{\partial n}
$$


According to the boundary element technique of Liao [67], we can extend the technique to deal with 3D dual-phase lag bioheat transfer equation by considering the following partial differential equations for $\Phi(\mathrm{x} ; \mathrm{P})$

$$
(1-\mathrm{P}) \mathrm{L}[\Phi(\mathrm{x} ; \mathrm{P})-\mathrm{U}(\mathrm{x})]=-\mathrm{PA}[\Phi(\mathrm{x} ; \mathrm{P})]
$$

with the boundary conditions

$$
\begin{aligned}
& x \in \Gamma_{1}: \Phi(x ; P)=P T_{b}^{f}(x)+(1-P) U(x) \\
& x \in \Gamma_{2}:-\widetilde{K} \frac{\partial \Phi(x ; P)}{\partial n}=P w_{b}^{f}(x)+(1-P)\left[-\widetilde{K} \frac{\partial U(x)}{\partial n}\right]
\end{aligned}
$$

The linear operator can be written as follows

$$
L(U)=\nabla^{2} u-B u=\frac{\partial^{2} u}{\partial x_{1}^{2}}+\frac{\partial^{2} u}{\partial x_{2}^{2}}+\frac{\partial^{2} u}{\partial x_{3}^{2}}-B u
$$

Using (11), the nonlinear operator can be written as

$$
A[\Phi(\mathrm{x} ; \mathrm{P})]=\nabla^{2} \Phi(\mathrm{x} ; \mathrm{P})-B \Phi(\mathrm{x} ; \mathrm{P})+C \nabla^{2} T^{f-1}(x)+D T^{f-1}+E T^{f-2}(x)+F
$$

If $\mathrm{P}=0$ then equations (13a) - (13c) can be expressed as

and

$$
L[\Phi(\mathrm{x} ; 0)=L[U(x)]]
$$

$$
\begin{aligned}
& \Phi(\mathrm{x} ; 0)=U(x) \text { for } x \in \Gamma_{1} \\
& -\widetilde{K} \frac{\partial \Phi(\mathrm{x} ; 0)}{\partial n}=-\widetilde{K} \frac{\partial U(x)}{\partial n} \text { for } x \in \Gamma_{2}
\end{aligned}
$$

It is clear that the solution of (15a) with boundary conditions (15b) and (15c) corresponds to the initial approximation $U(x)$ as

$\Phi(\mathrm{x} ; 0)=U(x)$

Also, if $\mathrm{P}=1$ then

$$
A[\Phi(\mathrm{x} ; 1)]=0
$$

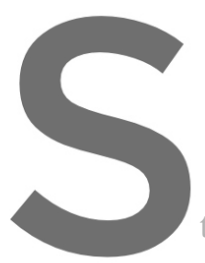

and

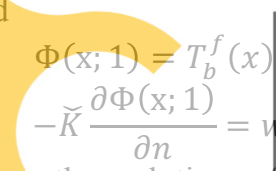

Also, the solution

temperature $T^{f}(x)$ as

$\Phi(\mathrm{x} ; 1)=T^{f}(x)$
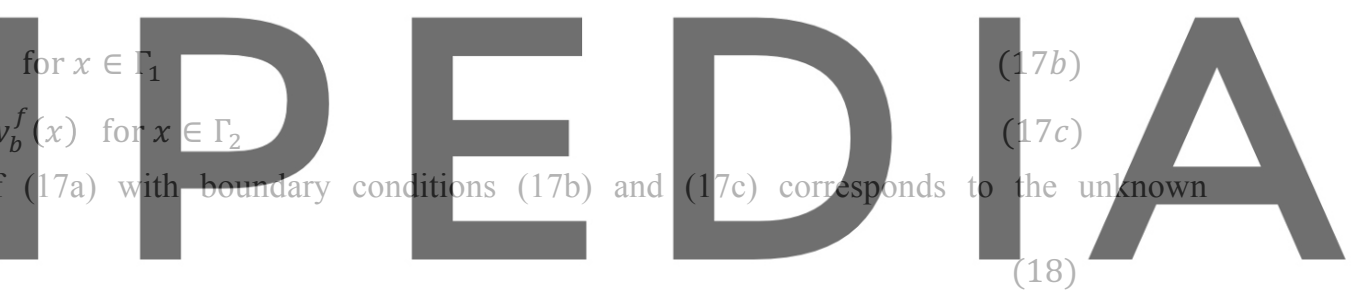

By differentiating equations (13a) - (13c) with respect to $\mathrm{P}$, we have

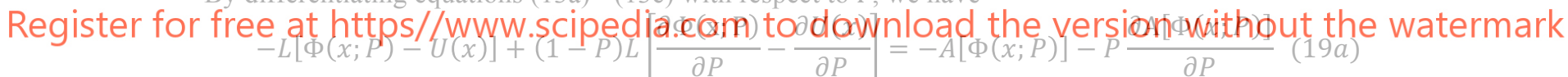

and

$$
\begin{aligned}
& \frac{\partial \Phi(\mathrm{x} ; \mathrm{P})}{\partial P}=T_{b}^{f}(x)+\frac{\partial T_{b}^{f}(x)}{\partial P}-U(x)+(1-P) \frac{\partial U(x)}{\partial P} \text { for } x \in \Gamma_{1} \\
& -\widetilde{K} \frac{\partial}{\partial n}\left(\frac{\partial \Phi(\mathrm{x} ; \mathrm{P})}{\partial P}\right)=w_{b}^{f}(x)+P \frac{\partial w_{b}^{f}(x)}{\partial P}+\widetilde{K} \frac{\partial U}{\partial n}-(1-P) \widetilde{K} \frac{\partial}{\partial n}\left[\frac{\partial U(x)}{\partial P}\right] \text { for } x \in \Gamma_{2}
\end{aligned}
$$

For $P=0$ and using (16) we have

$$
L\left[U^{[1]}(\mathrm{x})\right]=-\mathrm{A}[U(x)]
$$

and

$$
\begin{aligned}
& U^{[1]}(\mathrm{x})=T_{b}^{f}(x)-U(x) \text { for } x \in \Gamma_{1} \\
& -\widetilde{K}\left(\frac{\partial U^{[1]}(\mathrm{x})}{\partial n}\right)=w_{b}^{f}(x)+\widetilde{K} \frac{\partial U(x)}{\partial n} \text { for } x \in \Gamma_{2}
\end{aligned}
$$

where

$$
U^{[1]}(x)=\left.\frac{\partial \Phi(\mathrm{x} ; \mathrm{P})}{\partial P}\right|_{P=0}
$$

Making use of (14a) and (14b), we can write (20a) in the following form 
where

$$
\nabla^{2} U^{[1]}(x)-B U^{[1]}(x)+R[U(x)]=0
$$

$R[U(x)]=\nabla^{2} U(x)-B U(x)+C \nabla^{2} T^{f-1}(x)+D T^{f-1}(x)+E T^{f-2}(x)+F$

Applying Taylor series expansion to function $\Phi(\mathrm{x} ; \mathrm{P})$ about $P=0$ taking into account the first derivative

$$
\begin{aligned}
\Phi(\mathrm{x} ; \mathrm{P}) & =\Phi(\mathrm{x} ; \mathrm{P})+\left.\frac{\partial \Phi(\mathrm{x} ; \mathrm{P})}{\partial P}\right|_{P=0}(P-0) \\
& =\Phi(\mathrm{x} ; \mathrm{P})+U^{[1]}(x) P
\end{aligned}
$$

For $\mathrm{P}=1$, we obtain

$\Phi(\mathrm{x} ; 1)=\Phi(\mathrm{x} ; 0)+U^{[1]}(x)$

Using (16) and (18), this means

$T^{f}(x)=\mathrm{U}(\mathrm{x})+U^{[1]}(x)$

In order to obtain $U(x)$, we follow the following iterative rule [68]

$$
T_{k}^{f}(x)=T_{k-1}^{f}(x)+m U^{[1]}(x), \quad k=1,2,3, \ldots, K
$$

where $T_{0}^{f}(x)=T^{f-1}(x), m$ and $K$ are respectively iterative parameter and iterations number.

Now, we use the boundary element technique for each transition $\tau^{f-1} \rightarrow \tau^{f}$ to solve

$$
\nabla^{2} U^{[1]}(x)-B U^{[1]}(x)+R\left[T_{k-1}^{f}(x)\right]=0
$$

with boundary conditions (20b) and (20c) and then calculate the temperature using (22a)

For equation (22b), the corresponding boundary integral equation can be written as [62]

$$
B(\xi) U^{(1)}(\xi)-\int_{\Gamma} T^{*}(\xi, x) \frac{\partial U^{(1)}(x)}{\partial n} d \Gamma=-\int_{\Gamma} \frac{\partial T^{*}(\xi, x)}{\partial n} U^{(1)}(x) d \Gamma+\int_{\Omega} R\left[T_{k-1}^{f}(x)\right] T^{*}(\xi, x) d \Omega
$$

where, $B(\xi)(0<B(\xi)<1)$ is the point location - dependent coefficient, $\Gamma=\Gamma_{1} \cup \Gamma_{2}$.

Equation (23) can be written as
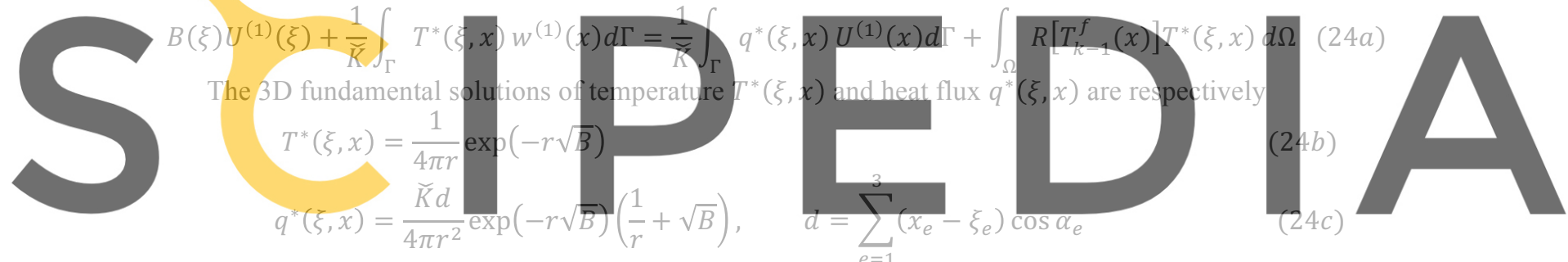

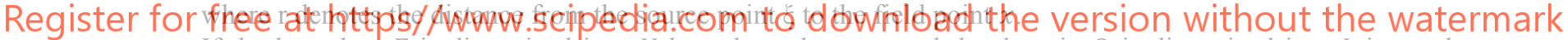

If the boundary $\Gamma$ is discretized into $N$ boundary elements and the domain $\Omega$ is discretized into $L$ internal elements, Eq. (24a) can be approximated as follows

where

$$
\sum_{j=1}^{i} G_{i j} w^{[1]}\left(x_{j}\right)=\sum_{j=1}^{i v} H_{i j} U^{[1]}\left(x_{j}\right)+\sum_{l=1}^{L} P_{i l} R\left[T_{k-1}^{f}\left(x_{l}\right)\right]
$$

$G_{i j}=\frac{1}{\breve{K}} \int_{\Gamma_{j}} T^{*}\left(\xi_{i}, x\right) d \Gamma_{j}, H_{i j}=\left\{\begin{array}{ll}\int_{\Gamma_{j}} q^{*}\left(\xi_{i}, x\right) d \Gamma_{j}, & i \neq j \\ -0.5, & i=j\end{array}, P_{i l}=\int_{\Omega_{j}} T^{*}\left(\xi_{i}, x\right) d \Omega_{j}\right.$

Using the boundary conditions (20b) and (20c) into (25), we obtain the unknowns $w^{(1)}$ and $U^{(1)}$ on the boundary. Then, the values $U^{(1)}\left(\xi_{i}\right)$ can be calculated as follows

$$
U^{(1)}\left(\xi_{i}\right)=\sum_{j=1}^{N} H_{i j} U^{(1)}\left(x_{j}\right)-\sum_{j=1}^{N} G_{i j} w^{(1)}\left(x_{j}\right)+\sum_{j=1}^{N} P_{i l} R\left[T_{k-1}^{f}\left(x_{l}\right)\right]
$$

Thus, with the temperature $T$ determined, the remaining task is to solve the poroelastic problem (8). 


\section{BOUNDARY ELEMENT IMPLEMENTATION FOR THE POROELASTIC FIELDS}

The representation formula for problem (8) which describes the unknown field $\hat{u}^{g}$ inside the domain is

$$
\hat{u}^{g}(\tilde{x})=\left(\widehat{V} \hat{t}^{g}\right)_{\Omega}(\tilde{x})-\left(\widehat{K} \hat{u}^{g}\right)_{\Omega}(\tilde{x}) \text { for } \tilde{x} \in \boldsymbol{\Omega}
$$

where the integral operators are

$$
\begin{aligned}
& \left(\hat{V} \hat{t}^{g}\right)_{\Omega}(\tilde{x})=\int_{\Gamma} \widehat{U}^{T}(y-\tilde{x}) \hat{t}^{g}(y) d s_{y} \\
& \left(\widehat{K} \hat{u}^{g}\right)_{\Omega}(\tilde{x})=\int_{\Gamma}\left(\hat{T}_{y} \widehat{U}\right)^{T}(y-\tilde{x}) \hat{u}^{g}(y) d s_{y}
\end{aligned}
$$

For anisotropic case, we used anisotropic fundamental solutions which proposed by Wang and Achenbach $[32,33]$, but for comparison purposes with other methods which are special cases of our general and complex study, we defined the Laplace domain fundamental solution $\widehat{U}(r)$ and the corresponding traction $\widehat{T}_{y}$ as [65].

$$
\widehat{U}(r)=\left[\begin{array}{ccc}
\widehat{U}^{s}(r) & \widehat{U}^{f}(r) & 0 \\
\left(\hat{P}^{s}\right)^{T}(r) & \widehat{P}^{f}(r) & 0
\end{array}\right], \widehat{T}_{y}=\left[\begin{array}{ccc}
T_{y}^{e} & s \alpha n_{y} & 0 \\
-\beta n_{y}^{T} & \frac{\beta}{s \rho^{f}} n_{y}^{T} \nabla & 0
\end{array}\right] \text { with } r:=|y-x|
$$

where the solid displacement fundamental solution $\widehat{U}^{s}(r)$ can be written as

$$
\widehat{U}^{s}(r)=\frac{1}{4 \pi r\left(\rho-\beta \rho^{f}\right)}\left[\mathbb{R}_{1} \frac{\left(k_{4}^{2}-k_{2}^{2}\right)}{\left(k_{1}^{2}-k_{2}^{2}\right)} e^{-k_{1} r}-\mathbb{R}_{2} \frac{\left(k_{4}^{2}-k_{1}^{2}\right)}{\left(k_{1}^{2}-k_{2}^{2}\right)} e^{-k_{2} r}+\left(I k_{3}^{2}-\mathbb{R}_{3}\right) e^{-k_{3} r}\right]
$$

with

$$
\mathbb{R}_{j}=\frac{3 \nabla_{y} r \nabla_{y}^{T} r-I}{r^{2}}+k_{j} \frac{3 \nabla_{y} r \nabla_{y}^{T} r-I}{r}+k_{j}^{2} \nabla_{y} r \nabla_{y}^{T} r
$$

which can be expressed as [61]

$$
\widehat{U}^{S}(r)=\frac{1}{4 \pi \mu r(\lambda+2 \mu)}[(\lambda+
$$

For the regularization, th

$\widehat{U}_{S}^{S}(r)$ and regular $\widehat{U}_{\mathrm{r}}^{S}(\mathrm{r})$ par
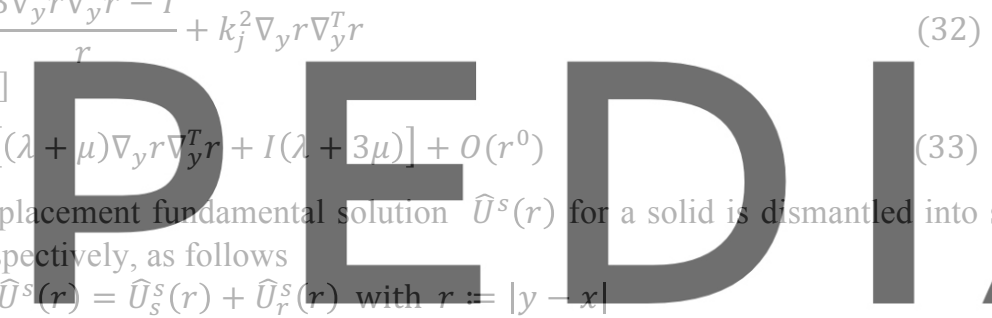

$\frac{1}{-}\left[I \Delta_{y}-\frac{\lambda+\mu}{n} \nabla_{y} \nabla_{\gamma}^{T}\right] \Delta_{y} \hat{x}(r)-\frac{1}{-}\left[\left(\left(k_{1}^{2}+k_{2}^{2}\right) \Delta_{y}-k_{1}^{2} k_{2}^{2}\right) J-\left(k_{1}^{2}+k_{2}^{2}-k_{4}^{2}-\frac{k_{1}^{2} k_{2}^{2}}{2}\right) \nabla_{y} \nabla_{y}^{T}\right] \hat{x}(r)$ (34) Register for free at hteps//WwW.scipedia.com to dowhload the version $k^{2}$ ithout the watermark where

$$
\begin{aligned}
\hat{x}(r)= & \frac{1}{4 \pi r}\left[\frac{e^{-k_{1} r}}{\left(k_{2}^{2}-k_{1}^{2}\right)\left(k_{3}^{2}-k_{1}^{2}\right)}+\frac{e^{-k_{2} r}}{\left(k_{2}^{2}-k_{1}^{2}\right)\left(k_{2}^{2}-k_{3}^{2}\right)}+\frac{e^{-k_{3} r}}{\left(k_{1}^{2}-k_{3}^{2}\right)\left(k_{2}^{2}-k_{3}^{2}\right)}\right] \\
& =-\frac{1}{\left(k_{1}^{2}-k_{2}^{2}\right)\left(k_{1}^{2}-k_{3}^{2}\right)\left(k_{3}^{2}-k_{2}^{2}\right)}+O\left(r^{2}\right)
\end{aligned}
$$

Also, the remaining parts of the fundamental solution $\widehat{U}(r)$ can be defined as

$$
\begin{aligned}
& \widehat{U}^{f}(r)=\frac{\rho^{f}(\alpha-\beta) \nabla_{y} r}{4 \pi r \beta(\lambda+2 \mu)\left(k_{1}^{2}-k_{2}^{2}\right)}\left[\left(k_{1}+\frac{1}{r}\right) e^{-k_{1} r}-\left(k_{2}+\frac{1}{r}\right) e^{-k_{2} r}\right]=O\left(r^{0}\right) \\
& \hat{P}^{s}(r)=\frac{\widehat{U}^{f}(r)}{s}=O\left(r^{0}\right) \\
& \hat{P}^{f}(r)=\frac{s \rho^{f}}{4 \pi r \beta\left(k_{1}^{2}-k_{2}^{2}\right)}\left[\left(k_{1}^{2}-k_{4}^{2}\right) e^{-k_{1} r}-\left(k_{2}^{2}-k_{4}^{2}\right) e^{-k_{2} r}\right]=\frac{s \rho^{f}}{4 \pi r \beta}+O\left(r^{0}\right)
\end{aligned}
$$

where $k_{1}, k_{2}, k_{3}$ and $k_{4}=k_{3} \sqrt{\frac{\mu}{\lambda+2 \mu}}$ are given in [61], $\widehat{U}_{s}^{s}(r)$ are of $O\left(r^{-1}\right)$ and $\widehat{U}_{r}^{s}(r)$ are of $O\left(r^{1}\right)$ where the following limiting process $\tilde{x} \in \Omega \rightarrow x \in \Gamma$ is performed on (28) to obtain

$$
\lim _{\tilde{x} \in \Omega \rightarrow x \in \Gamma}\left(\hat{V} \hat{t}^{g}\right)_{\Omega}(\tilde{x})=\left(\hat{V} \hat{x}^{g}\right)(x):=\int_{\Gamma} \widehat{U}^{T}(y-x) \hat{t}^{g}(y) d s_{y}
$$


Also, we perform the following limiting process $\tilde{x} \in \Omega \rightarrow x \in \Gamma$ on (29) to have [67]

$$
\lim _{\tilde{x} \in \Omega \rightarrow x \in \Gamma}\left(\widehat{K} \hat{u}^{g}\right)_{\Omega}(\tilde{x})=[-I(x)+C(x)] \hat{u}^{g}(x)+\left(\widehat{K} \hat{u}^{g}\right)(x)
$$

where

$$
C(x)=\lim _{\varepsilon \rightarrow 0} \int_{y \in \Omega:|y-x|=\varepsilon}\left(\widehat{T}_{y} \widehat{U}\right)^{T}(y-x) d s_{y}
$$

where the double layer operator is

$$
\left(\widehat{K} \hat{u}^{g}\right)(x)=\lim _{\varepsilon \rightarrow 0} \int_{|y-x| \geq \varepsilon}\left(\widehat{T}_{y} \widehat{U}\right)^{T}(y-x) \hat{u}^{g}(y) d s_{y}
$$

Using equations (39) - (42), the Laplace domain boundary integral equation can be expressed as

$$
C(x) \hat{u}^{g}(x)=\left(\widehat{V} \hat{t}^{g}\right)(x)-\left(\widehat{K} \hat{u}^{g}\right)(x)
$$

Applying inverse Laplace transformation to obtain the following boundary integral equation

$$
C(x) u^{g}(x, t)=\left(V * t^{g}\right)(x, t)-\left(K u^{g}\right)(x, t)
$$

According to [65] The poroelastodynamic fundamental solution can be expressed as follows

$$
\left(\widehat{T}_{y} \widehat{U}\right)^{T}=\left[\left[\begin{array}{cc}
\widehat{T}_{y}^{e} & \operatorname{san}_{y} \\
-\beta n_{y}^{T} & \frac{\beta}{s \rho_{0}^{f}} n_{y}^{T} \nabla_{y}
\end{array}\right]\left[\begin{array}{cc}
\widehat{U}^{S} & \widehat{U}^{f} \\
\left(\hat{P}^{S}\right)^{T} & \hat{P}^{f}
\end{array}\right]\right]^{T}=\left[\begin{array}{cc}
\widehat{T}^{S} & \widehat{T}^{f} \\
\left(\hat{Q}^{S}\right)^{T} & \hat{Q}^{f}
\end{array}\right]^{T}
$$

According to Stokes theorem, the differentiable vector field a $(y)$ with $y \in \Gamma$ can be written as

$$
\int_{\Gamma}\left(\nabla_{y} \times \mathrm{a}, n_{y}\right) d s_{y}=-\int_{\partial \Gamma}(\mathrm{a}, v) d \gamma_{y}=-\int_{\phi}(\mathrm{a}, v) d \gamma_{y}=0
$$
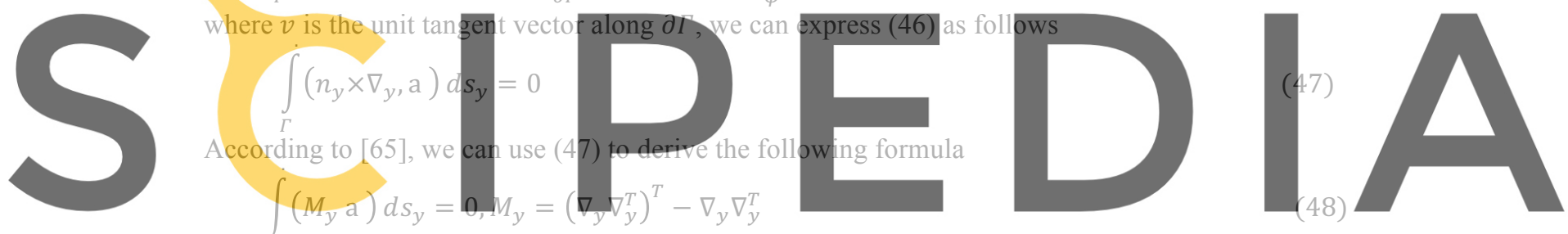

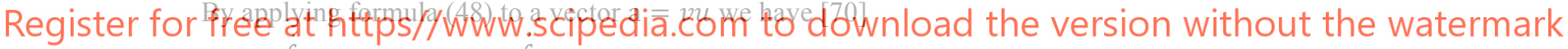

$$
\begin{aligned}
& \int_{\Gamma}\left(M_{y} v\right) u d s_{y}=-\int_{\Gamma} v\left(M_{y} u\right) d s_{y} \\
& \int_{\Gamma}^{\Gamma_{0}}\left(M_{y} v\right)^{T} u d s_{y}=\int_{\Gamma} v^{T}\left(M_{y} u\right) d s_{y}
\end{aligned}
$$

Using (34) and (45), we can write $\widehat{T}^{s}$ as follows

$$
\left(\widehat{T}^{s}\right)^{T}=\left(T_{y}^{e}\left(\widehat{U}_{\text {sing }}^{s}+\widehat{U}_{\text {reg }}^{s}\right)\right)^{T}+s \alpha \widehat{P}^{s} n_{y}^{T}=\left(T_{y}^{e} \widehat{U}_{\text {sing }}^{s}\right)^{T}+O\left(r^{0}\right)
$$

Using the same representation of $T_{y}^{e}$ as in [65], we can write

$$
\left(\widehat{T}^{s}\right)^{T}=(\lambda+2 \mu) n_{y} \nabla_{y}^{T} \widehat{U}_{\text {sing }}^{s}-\mu\left(n_{y} \times\left(\nabla_{y} \times \widehat{U}_{\text {sing }}^{s}\right)\right)+2 \mu M_{y} \hat{u}_{\text {sing }}^{s}+o\left(r^{0}\right)
$$

which can be written using (34) in the form

$$
\left(\widehat{T}^{s}\right)^{T}=M_{y} \Delta_{y}^{2} \hat{X}+I\left(\mathrm{n}^{T} \nabla_{y}\right) \Delta_{y}^{2} \hat{X}+2 \mu\left(M_{y} \widehat{U}_{\text {sing }}^{s}\right)^{T}+o\left(r^{0}\right)
$$

Applying definition (29) to Eq. (53), we get

$$
(\hat{k} \hat{u})_{\Omega}^{s}(\tilde{x})=\int_{\Gamma}\left[\left(M_{y} \Delta_{y}^{2} \hat{X}\right) \hat{u}+\left(I\left(n^{T} \nabla_{y}\right) \Delta_{y}^{2} \hat{X}\right) \hat{u}+2 \mu\left(M_{y} \widehat{U}_{\text {sing }}^{s}\right)^{T} \hat{u}+O\left(r^{0}\right) \hat{u}\right] d s_{y}
$$

Applying (49) to the first term and applying (50) to the third term on the right-hand side of (54), also, making use of the Duffy transformation and a standard Gaussian quadrature rule, we obtain [69] 


$$
(\widehat{K} \hat{u})_{\Omega}^{s}(\tilde{x})=\int_{\Gamma}\left[-\Delta_{y}^{2} \hat{X}\left(M_{y} \hat{u}\right)+\left(I\left(n^{T} \nabla_{y}\right) \Delta_{y}^{2} \hat{x}\right) \hat{u}+2 \mu \widehat{U}_{s}^{s}\left(M_{y} \hat{u}\right)+O\left(r^{0}\right) \hat{u}\right] d s_{y}
$$

The second term in the right side of (55) has been manipulated to get

where

$$
\left(n^{T} \nabla_{y}\right) \Delta_{y}^{2} \hat{x}(r)=\frac{n^{T} \nabla_{y} r}{4 \pi r^{2}}+O\left(r^{0}\right)
$$

$$
C^{s}(x)=I(x) c(x) \text { with } c(x)=\frac{\phi(x)}{4 \pi}
$$

According to [65], the solid related part of the limit (32) can be reexpressed as follows

$$
\lim _{\Omega \ni \tilde{x} \rightarrow x \in \Gamma}(\widehat{K} \hat{u})_{\Omega}^{s}(\tilde{x})=-I(x)[-1+c(x)] \hat{u}(x)+(\widehat{K} \hat{u})^{S}(x)
$$

By augmenting $\widehat{U}_{s}^{s}$ to $\widehat{U}^{s}$ and using (50) we obtain a more efficient form of (55) as follows

$$
(\widehat{K} \hat{u})_{\Omega}^{S}(\tilde{x})=\int_{\Gamma}-\Delta_{y}^{2} \hat{x}\left(M_{y} \hat{u}\right)+\left(I\left(n^{T} \nabla_{y}\right) \Delta_{y}^{2} \hat{x}\right) \hat{u}+2 \mu \widehat{U}^{S}\left(M_{y} \hat{u}\right)+O\left(r^{0}\right) \hat{u} d s_{y}
$$

Discretizing the time interval $0 \leq t \leq T$ into $N+1$ equal time stens $\Delta t>0$ with discrete times $t_{n}=n \Delta t$

The convolution quadrature method numerically approximates the following convolution integral

$$
(f * g)(t)=\int_{0}^{t} f(t-\tau) g(\tau) d \tau \text { for } t \in[0, T]
$$

by the finite sum

$$
(f * g)\left(t_{n}\right) \approx \sum_{k=0}^{n} \omega_{n-k}^{\Delta t}(\hat{f}) g\left(t_{k}\right)
$$

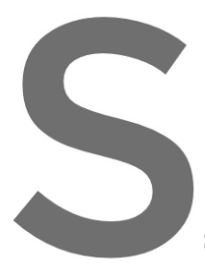

The integration weigh
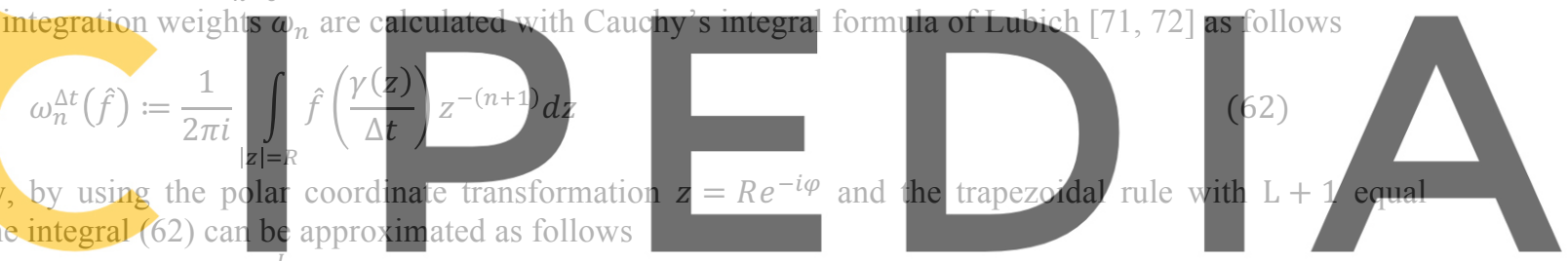

Register for free at https//WwW.Scipedia.com to download the version without the watermark By substituting Eq. (63) into Eq. (61), we obtain

wiith

$$
(f * g)\left(t_{n}\right) \approx \sum_{k=0}^{N} \frac{R^{-(n-k)}}{N+1} \sum_{\ell=0}^{N} \hat{f}\left(s_{\ell}\right) \zeta^{\ell(n-k)} g\left(t_{k}\right) \approx \frac{R^{-n}}{N+1} \sum_{\ell=0}^{N} \hat{f}\left(s_{\ell}\right) \hat{g}\left(s_{\ell}\right) \zeta^{\ell n}
$$

$$
\hat{g}\left(s_{\ell}\right)=\sum_{k=0}^{N} R^{k} g\left(t_{k}\right) \zeta^{-\ell k}
$$

By applying the procedure [70] to the convolution operator of our problem (44), we obtain

$$
C(x) u^{g}(x, t)=\left(v * t^{g}\right)(x, t)-\left(k * u^{g}\right)(x, t)
$$

which can be expressed as

$$
C(x) \hat{u}^{g}\left(x, s_{\ell}\right)=\left(\hat{v} \hat{t}^{g}\right)\left(x, s_{\ell}\right)-\left(\hat{k} \hat{u}^{g}\right)\left(x, s_{\ell}\right), \quad \ell=0 \ldots \ldots . . N
$$

Let the boundary $\Gamma=\partial \Omega$ is discretized into $N_{e}$ surface triangles boundary elements $\tau_{e}$ as (Fig. 2a)

$$
\Gamma \approx \Gamma_{h}=\bigcup_{e=1}^{N_{e}} \tau_{e}
$$


Now, we define the following subspaces on $\Gamma_{h}$ as

$$
\begin{aligned}
& S_{h}[k]\left(\Gamma_{N, h}\right):=\operatorname{span}\left\{\varphi_{i}^{\alpha}[k]\right\}_{i=1}^{\stackrel{\sharp}{1}}, \quad \alpha \geq 1 \\
& S_{h}[k]\left(\Gamma_{D, h}\right):=\operatorname{span}\left\{\psi_{j}^{\beta}[k]\right\}_{j=1}^{\mathfrak{D}}, \quad \beta \geq 0
\end{aligned}
$$

where the unknown Neumann datum is approximated with if continuous polynomial shape functions $\varphi_{i}^{\alpha}[k]$ and time dependent coefficients. Also, the unknown Dirichlet datum is approximated with piecewise discontinuous polynomial shape functions $\psi_{j}^{\beta}[k]$ and time dependent coefficients as follows

$$
\begin{aligned}
& \hat{u}^{g}[k](x) \approx \hat{u}_{h}^{g}[k](x)=\sum_{i=1}^{\mathbb{I}} \hat{u}_{h, i}^{g}[k] \varphi_{i}^{\alpha}[k](x) \in S_{h}[k]\left(\Gamma_{N, h}\right) \\
& \hat{t}^{g}[k](x) \approx \hat{t}_{h}^{g}[k](x)=\sum_{j=1}^{\mathbb{j}} \hat{t}_{h, j}^{g}[k] \psi_{j}^{\beta}[k](x) \in S_{h}[k]\left(\Gamma_{D, h}\right)
\end{aligned}
$$

where $k=1,2,3,4$ are the poroelastic degrees of freedom, $i=1, \ldots, I$ are the collocation points on the on the Neuman boundary and $j=1, \ldots, J$ are the collocation points on the Dirichlet boundary.

Inserting these spatial shape functions into (67), yields the following $N+1$ algebraic systems of equations

$$
\left[\begin{array}{l}
\widehat{V}_{D D}-\widehat{K}_{D N} \\
\widehat{V}_{N D}-\left(C+\widehat{K}_{N N}\right)
\end{array}\right]_{\ell}\left[\begin{array}{l}
\hat{t}_{D, h}^{g} \\
\hat{u}_{N, h}^{g}
\end{array}\right]_{\ell}=\left[\begin{array}{ll}
-\widehat{V}_{D N} & \left(C+\widehat{K}_{D D}\right) \\
-\widehat{V}_{N N} & \widehat{K}_{N D}
\end{array}\right]_{\ell}\left[\begin{array}{l}
\hat{g}_{N, h}^{g} \\
\hat{g}_{D, h}^{g}
\end{array}\right]_{\ell} \ell=0 \ldots N
$$

where the Schur complement of the block of the system matrix can be defined as follows

$$
\hat{S}_{N N}:=\widehat{V}_{N D} \widehat{V}_{D D}^{-1} \widehat{K}_{D N}-\left(C+\widehat{K}_{N N}\right)
$$

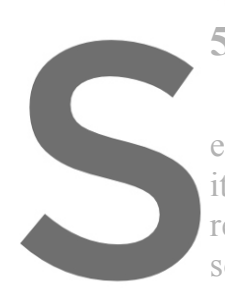

5 NUMERICAL RESU

\section{Since the system of equatic} et al. [73] investigated that

iterations number and CPU

residual (GMRES) algorithn

solve the resulting linear sy
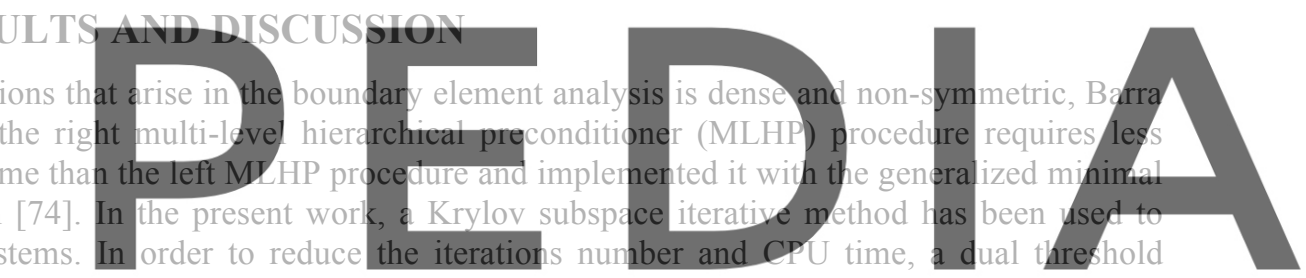

incomplete LU factorization technique (ILUT) which is one of the well-known preconditioning techniques is

Register implemented as a robust preconditioner for TFQMR (Transpose-free quasi minimal residual) [75], to accelerate

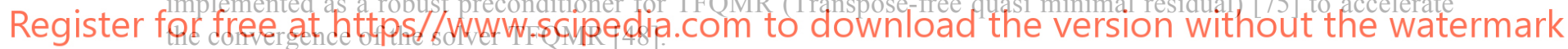

The specific absorption rate can be expressed as [76]

$$
Q_{r}=S_{0} P_{0}(\tau) e^{-S_{0} x}
$$

In order to explain the calculations of the proposed technique, we used the following transversely isotropic soft tissue parameters [77]

The elasticity tensor

$$
\begin{aligned}
C_{a b l g}= & {\left[\begin{array}{cccccc}
C_{11} & C_{12} & C_{13} & 0 & 0 & 0 \\
C_{12} & C_{11} & C_{13} & 0 & 0 & 0 \\
C_{13} & C_{13} & C_{33} & 0 & 0 & 0 \\
0 & 0 & 0 & C_{44} & 0 & 0 \\
0 & 0 & 0 & 0 & C_{44} & 0 \\
0 & 0 & 0 & 0 & 0 & C_{66}
\end{array}\right] } \\
& C_{11}=\frac{E^{2} v_{0}^{2}-E E_{0}}{(1+v)\left(2 E v_{0}^{2}+E_{0}(v-1)\right)}, C_{12}=-\frac{E^{2} v_{0}^{2}+E E_{0} v}{(1+v)\left(2 E v_{0}^{2}+E_{0}(v-1)\right)} \\
C_{13}= & -\frac{E E_{0} v}{2 E v_{0}^{2}+E_{0}(v-1)}, C_{33}=-\frac{E_{0}^{2}(v-1)}{2 E v_{0}^{2}+E_{0}(v-1)}, C_{44}=\mu_{0}, C_{66}=\frac{1}{2}\left(C_{11}-C_{12}\right)
\end{aligned}
$$

where

$v=0.196, v_{0}=0.163, \mu_{0}=20.98 \mathrm{GPa}, E=68.34 \mathrm{GPa}, E_{0}=51.35 \mathrm{GPa}, k_{1}=108.39 \mathrm{GPa}, k_{2}=-21.70 \mathrm{GPa}$

where $E$ and $E_{0}$ are the Young's moduli in the isotropy plane and fiber direction respectively, $v$ and $v_{0}$ are Poisson's ratio in the isotropy plane and fiber direction respectively, and $\mu_{0}$ is the shear moduli in the plane 
perpendicular to isotropy plane.

We used the following strongly anisotropic soft tissue parameters [78]

$$
\mathrm{v}=0.95, \mathrm{v}_{0}=0.49, \mu_{0}=20.98 \mathrm{GPa}, \mathrm{E}=22 \mathrm{kPa}, \mathrm{E}_{0}=447 \mathrm{kPa}, \mathrm{k}_{1}=1243 \mathrm{kPa}, \mathrm{k}_{2}=442 \mathrm{kPa}
$$

and other constants taking into consideration are as follows

$\rho_{s}=1600 \mathrm{~kg} / \mathrm{m}^{3}, \rho_{\mathcal{F}}=1113 \mathrm{~kg} / \mathrm{m}^{3}, \mathrm{p}=25 \mathrm{MPa}, \phi=0.15$ and $\mathrm{Q} / \mathrm{R}=0.65$.

In the CQBEM modelling of the considered problem, the boundary has been discretized using 84 linear boundary elements and 404 internal points as shown in Fig. 1b. The computation was done using Matlab R2018a on a MacBook Pro with $2.9 \mathrm{GHz}$ quad-core Intel Core i7 processor and 16GB RAM.

The results of $1 \mathrm{~T}$ and $2 \mathrm{~T}$ models are presented graphically in figures 2-4 which show the variation of the displacements $u_{1}, u_{2}$ and $u_{3}$ with the time $\tau$ for the CQBEM and analytical solution of [79]. It can be seen from these figures that the results of $2 \mathrm{~T}$ model show more good agreement than the results of $1 \mathrm{~T}$ model. The difference between analytical and numerical results due to anisotropy properties of the biological tissues considered in the CQBEM. It can be seen from these figures that the CQBEM results are in good agreement with the analytical results. Our results thus confirm that our method is efficient and accurate.
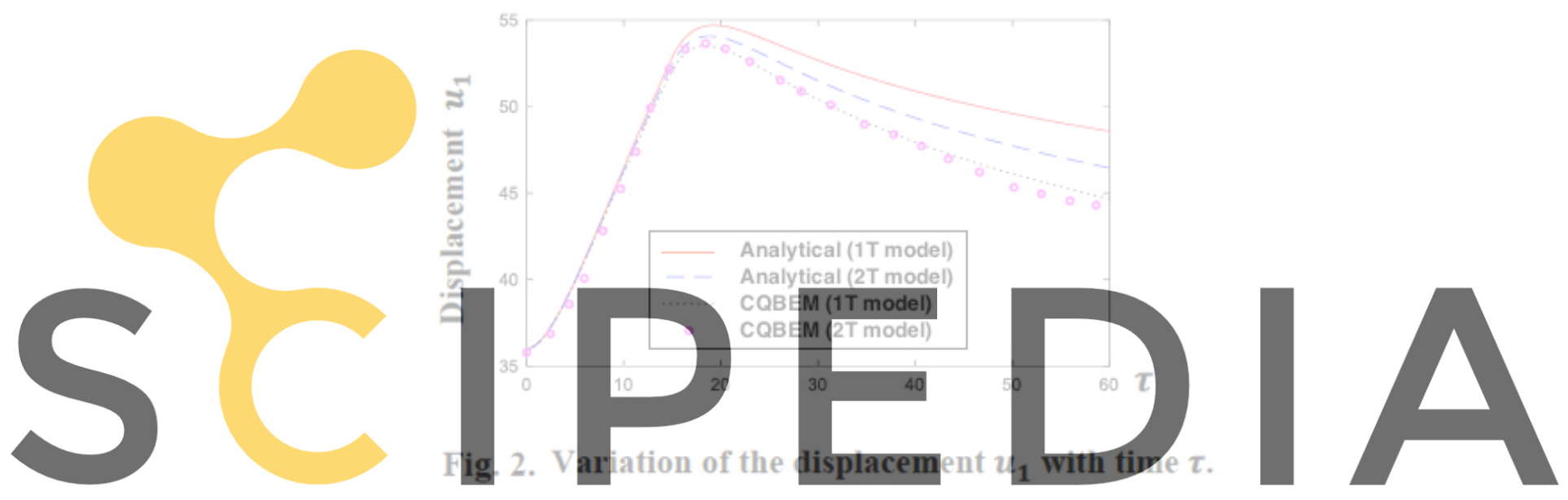

Register for free at https//www.scipedia.com to download the version without the watermark

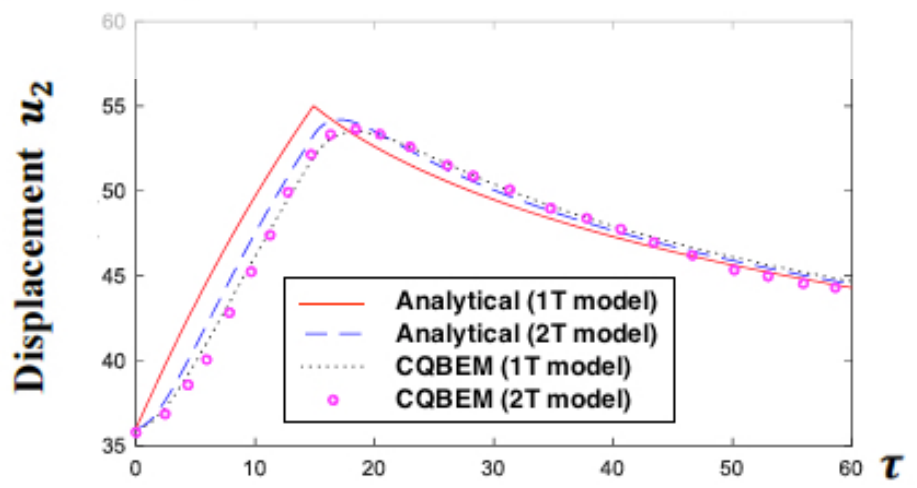

Fig. 3. Variation of the displacement $u_{2}$ with time $\tau$. 


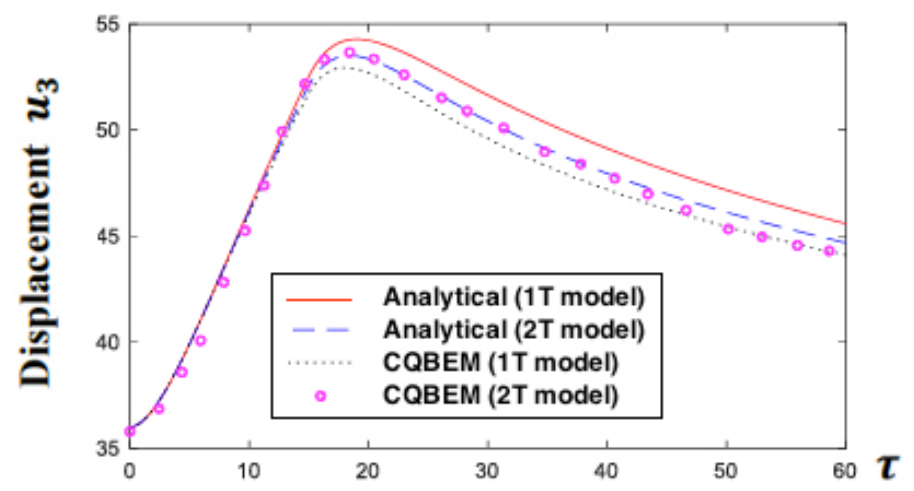

Fig. 4. Variation of the displacement $u_{3}$ with time $\tau$.

\section{CONCLUSIONS}

The main conclusion of this paper is to develop a new boundary element technique for describing the biothermomechanical interactions in anisotropic biological tissues. The uncoupled governing equations are resolved independently, Where the dual phase lag bioheat transfer equation is resolved first for one-temperature and two-temperature models to obtain the temperature distribution and then the displacement distributions are obtained by solving the mechanical equation using the proposed CQBEM, which is a flexible and efficient method, since it deals with more complex shapes of biological tissues and does not involve discretization, also, it has low RAM and CPU usage. The resulting linear equations arising from CQBEM solution of bioheat and mechanical equations are solved by the GMSS method which reduces the iterations number and total CPU time. Numerical findings demonstrate the validity, efficacy and consistency of the proposed technique.

\section{REFERENCES}

[1] Cho, Y.I. Advances in Heat Transfer: Bioengineering Heat Transfer. Academic Press Inc., Vol. 22, (1992).

[2] Pennes, H.H. Analysis of tissue and arterial blood temperatures in the resting human forearm, Journal of Applied Physiology (1948) 1:93-122,.

[3] Askarizadeh, H. and Ahmadikia H. Analytical study on the transient heating of a two-dimensional skin tissue using parabolic and hyperbolic bioheat transfer equations, Applied Mathematical Modelling (2015) 39:3704-3720.

[4] Li, X., Li, C., Xue, Z. and Tian, X. Analytical study of transient thermo-mechanical responses of dual-layer skin tissue with variable thermal material properties, International Journal of Thermal Sciences (2018) 124:459-466.

[5] Ahmadikia H., Fazlali, R. and Moradi A. Analytical solution of the parabolic and hyperbolic heat transfer equations with constant and transient heat flux conditions on skin tissue, International Communications in Heat and Mass Transfer (2012) 39:121-130.

[6] Shih, T.C., Yuan P., Lin, W.L. and Kou H.S. Analytical analysis of the Pennes bioheat transfer equation with sinusoidal heat flux condition on skin surface, Medical Engineering and Physics (2007) 29:946-953.

[7] El-Naggar, A.M., Abd-Alla, A.M., Fahmy, M.A. and Ahmed, S.M. Thermal Stresses in a rotating nonhomogeneous orthotropic hollow cylinder. Heat and Mass Transfer (2002) 39, pp. 41-46.

[8] Abd-Alla, A.M., El-Naggar, A.M. and Fahmy, M.A. Magneto-thermoelastic problem in non-homogeneous isotropic cylinder. Heat and Mass Transfer (2003) 39:625-629.

[9] El-Naggar, A.M., Abd-Alla, A.M. and Fahmy, M.A. The propagation of thermal stresses in an infinite elastic slab, Applied Mathematics and Computation (2003) 12:220-226. 
[10] Eric Li, G. R. Liu, Vincent Tan, Z. C. He, Modeling and simulation of bioheat transfer in the human eye using the $3 \mathrm{D}$ alpha finite element method $(\alpha \mathrm{FEM})$, International journal for numerical Methods in Biomedical Engineering (2010) 26:955-976.

[11] Abd-Alla, A.M., Fahmy, M.A. and El-Shahat, T.M. Magneto-thermo-elastic problem of a rotating nonhomogeneous anisotropic solid cylinder. Archive of Applied Mechanics (2008) 78:135-148.

[12] Fahmy, M.A. Thermoelastic stresses in a rotating non- homogeneous anisotropic body. Numerical Heat Transfer, Part A: Applications (2008) 53:1001-1011.

[13] Fahmy, M.A. and El-Shahat, T.M. The effect of initial stress and inhomogeneity on the thermoelastic stresses in a rotating anisotropic solid. Archive of Applied Mechanics (2008) 78:431-442.

[14] Fahmy, M.A. and Salama, S.A. Boundary element solution of steady-state temperature distribution in nonhomogeneous media. Far East Journal of Applied Mathematics (2010) 43:31-40.

[15] Fahmy, M.A. Application of DRBEM to non steady-state heat conduction in non-homogeneous anisotropic media under various boundary elements. Far East Journal of Mathematical Sciences (2010) 43:83-93.

[16] Fahmy, M.A. A time-stepping DRBEM for magneto-thermo-viscoelastic interactions in a rotating nonhomogeneous anisotropic solid. International Journal of Applied Mechanics (2011) 3:1-24.

[17] Fahmy, M.A. A time-stepping DRBEM for the transient magneto-thermo-visco-elastic stresses in a rotating non-homogeneous anisotropic solid. Engineering Analysis with Boundary Elements (2012) 36:335-345.

[18] Fahmy, M.A. Transient magneto-thermoviscoelastic plane waves in a non-homogeneous anisotropic thick strip subjected to a moving heat source. Applied Mathematical Modelling (2012) 36:4565-4578.

[19] Fahmy, M.A. Numerical modeling of transient magneto-thermo-viscoelastic waves in a rotating nonhomogeneous anisotropic solid under initial stress. International Journal of Modeling, Simulation and Scientific Computing (2012) 3:1250002

[20] Fahmy, M.A. The effect of rotation and inhomogeneity on the transient magneto-thermoviscoelastic stresses in an anisotropic solid. ASME Journal of Applied Mechanics (2012) 79:1015.

[21] Fahmy, M.A. Transient magneto-thermo-viscoelastic stresses in a rotating nonhomogeneous anisotropic solid with and without a moving heat source, Journal of Engineering Physics and Thermophysics (2012) 85:950-958.

[22] Pei, R.Z., Jing, L., Cheng, W.C., Xue, J.P. Boundary element method (BEM) for solving normal or inverse bio-heat transfer problem of biological bodies with complex shape. Journal of Thermal Science (1995) 4:117-124.

[23] Ooi, E.H., Ang, W.T. and Ng, E.Y.K. A boundary element model for investigating the effects of eye tumor on the temperature distribution inside the human eye, Computers in Biology and Medicine (2009) 39:667677.

[24] Zhou, J., Chen, J.K., and Zhang Y., Simulation of Laser-Induced Thermotherapy Using a Dual-Reciprocity Boundary Element Model with Dynamic Tissue Properties. IEEE Transactions on Biomedical Engineering (2010) 57:238-245.

[25] Ng E.Y.K., Tan H.M. and Ooi E.H. Boundary element method with bioheat equation for skin burn injury. Burns (2009) 35:987 - 997.

[26] Majchrzak, E., Turchan L. The general boundary element method for 3D dual-phase lag model of bioheat transfer, Engineering Analysis with Boundary Elements. Engineering Analysis with Boundary Elements (2015) 50:76-82.

[27] Bottauscio, O., Chiampi, M. and Zilberti, L. Boundary Element Solution of Electromagnetic and Bioheat Equations for the Simulation of SAR and Temperature Increase in Biological Tissues. IEEE Transactions on Magnetics (2012) 48:691-694.

[28] Deng Z.S. and Liu J. Modeling of multidimensional freezing problem during cryosurgery by the dual reciprocity boundary element method. Engineering Analysis with Boundary Elements (2004) 28:97-108.

[29] Partridge P.W. and Wrobel L.C. A coupled dual reciprocity BEM/genetic algorithm for identification of blood perfusion parameters. International Journal of Numerical Methods for Heat \& Fluid Flow (2009) 19:25-38.

[30] Chan C.L. Boundary Element Method Analysis for the Bioheat Transfer Equation. Journal of Biomechanical Engineering (1992) 114:358-365.

[31] Wrobel L.C. The boundary element method, Applications in thermos-fluids and acoustics, Wiley, Vol. 1, 
(2002).

[32] Fahmy, M.A. Transient magneto-thermo-elastic stresses in an anisotropic viscoelastic solid with and without moving heat source. Numerical Heat Transfer, Part A: Applications (2012) 61:547-564

[33] Fahmy, M.A. Implicit-Explicit time integration DRBEM for generalized magneto-thermoelasticity problems of rotating anisotropic viscoelastic functionally graded solids. Engineering Analysis with Boundary Elements (2013) 37:107-115.

[34] Fahmy, M.A. Generalized magneto-thermo-viscoelastic problems of rotating functionally graded anisotropic plates by the dual reciprocity boundary element method. Journal of Thermal Stresses (2013) 36:1-20.

[35] Fahmy, M.A. A three-dimensional generalized magneto-thermo-viscoelastic problem of a rotating functionally graded anisotropic solids with and without energy dissipation. Numerical Heat Transfer, Part A: Applications (2013) 63:713-733.

[36] Fahmy, M.A. A 2-D DRBEM for generalized magneto-thermo-viscoelastic transient response of rotating functionally graded anisotropic thick strip. International Journal of Engineering and Technology Innovation (2013) 3:70-85.

[37] Fahmy, M.A. The DRBEM solution of the generalized magneto-thermo-viscoelastic problems in 3D anisotropic functionally graded solids. Idelsohn, I., Papadrakakis, M. and Schrefler, B. (eds.), Proceedings of the 5th International conference on coupled problems in science and engineering (Coupled Problems 2013), Ibiza, Spain, June 17-19, pp. 862-872.

[38] Fahmy, M.A. A Computerized DRBEM model for generalized magneto-thermo-visco-elastic stress waves in functionally graded anisotropic thin film/substrate structures. Latin American Journal of Solids and Structures (2014) 11:386-409.

[39] Fahmy, M.A. A 2D time domain DRBEM computer model for magneto-thermoelastic coupled wave propagation problems. International Journal of Engineering and Technology Innovation (2014) 4:138-151.

[40] Fahmy, M.A. Boundary Element Solution of 2D Coupled Problem in Anisotropic Piezoelectric FGM Plates. Schrefler, B., Oñate, E. and Papadrakakis, M. (eds.), Proceedings of the 6th International Conference on Computational Methods for Coupled Problems in Science and Engineering (Coupled Problems 2015), Venice. Italy, May 18-20, pp. 382-391.

[41] Fahmy, M.A. 3D DRBEM Modeling for Rotating initially stressed Anisotropic functionally graded piezoelectric Plates, Proceedings of the 7th European Congress on Computational Methods in Applied Sciences and Engineering (ECCOMAS 2016) Papadrakakis, M., Papadopoulos, V., Stefanou, G. and Plevris, V. (eds.), Crete Island, Greece, June 5-10, pp. 7640-7658.

[42] Fahmy, M.A. (2017), A Predictor-corrector Time-Stepping Drbem for Shape Design Sensitivity and Optimization of Multilayer FGA Structures, Transylvanian Review, Vol. XXV, pp. 5369-5382.

[43] [43] Fahmy, M.A. (2017), Computerized boundary element solutions for thermoelastic problems: Applications to functionally graded anisotropic structures, LAP Lambert Academic Publishing, Vol. 1, (2017).

[44] Fahmy, M.A. (2017), Boundary Element Computation of Shape Sensitivity and Optimization: Applications to Functionally Graded Anisotropic Structures, LAP Lambert Academic Publishing, Vol. 1, (2017).

[45] Fahmy, M.A. Shape design sensitivity and optimization for two-temperature generalized magnetothermoelastic problems using time-domain DRBEM. Journal of Thermal Stresses (2018) 41:119-138.

[46] Fahmy, M.A. Shape design sensitivity and optimization of anisotropic functionally graded smart structures using bicubic B-splines DRBEM. Engineering Analysis with Boundary Elements (2018) 87:27-35.

[47] Fahmy, M.A. A New Computerized Boundary Element Algorithm for Cancer Modeling of Cardiac Anisotropy on the ECG Simulation. Asian Journal of Research in Computer Science (2018) 2:1-10.

[48] Fahmy, M.A. Boundary Element Algorithm for Modeling and Simulation of Dual Phase Lag Bioheat Transfer and Biomechanics of Anisotropic Soft Tissues. International Journal of Applied Mechanics (2018) 10:1850108.

[49] Fahmy, M.A. Modeling and Optimization of Anisotropic Viscoelastic Porous Structures Using CQBEM and Moving Asymptotes Algorithm. Arabian Journal for Science and Engineering (2019) 44:1671-1684.

[50] Fahmy, M.A. Boundary Element Modeling and Simulation of Biothermomechanical Behavior in Anisotropic Laser-Induced Tissue Hyperthermia. Engineering Analysis with Boundary Elements (2019) 
101:156-164.

[51] Fahmy, M.A. A new LRBFCM-GBEM modeling algorithm for general solution of time fractional order dual phase lag bioheat transfer problems in functionally graded tissues. Numerical Heat Transfer, Part A: Applications (2019) 75:616-626.

[52] Fahmy, M.A. Design Optimization for A Simulation of Rotating Anisotropic Viscoelastic Porous Structures Using Time-Domain OQBEM. Mathematics and Computers in Simulation (2019) 66, 193-205.

[53] Fahmy, M.A. A Computerized Boundary Element Model for Simulation and Optimization of FractionalOrder Three Temperatures Nonlinear Generalized Piezothermoelastic Problems Based on Genetic Algorithm. AIP Conference Proceedings (2019) 2138:030015.

[54] Fahmy, M.A. A New Boundary Element Strategy for Modeling and Simulation of Three Temperatures Nonlinear Generalized Micropolar-Magneto-Thermoelastic Wave Propagation Problems in FGA Structures. Engineering Analysis with Boundary Elements (2019) 108:192-200.

[55] Fahmy, M.A. A new convolution variational boundary element technique for design sensitivity analysis and topology optimization of anisotropic thermo-poroelastic structures. Arab Journal of Basic and Applied Sciences (2020) 27:1-12.

[56] Fahmy, M.A. Boundary element algorithm for nonlinear modeling and simulation of three temperature anisotropic generalized micropolar piezothermoelasticity with memory-dependent derivative. International Journal of Applied Mechanics (2020) 12:2050027.

[57] Wang C.Y. and Achenbach J.D. Elastodynamic fundamental solution for anisotropic solids. Geophysical Journal International (1994) 118:384-392.

[58] Wang, C.Y. and Achenbach J.D. Three-dimensional time-harmonic elastodynamic Green's functions for anisotropic solids, Proceedings of the Royal Society A: Mathematical, Physical and Engineering Sciences (1995) 449:441-458.

[59] Biot, M.A. Theory of propagation of elastic waves in a fluid- saturated porous solid. I. Low frequency range. The Journal of the Acoustical Society of America (1956) 28:168-178.

[60] Biot M.A. Theory of propagation of elastic waves in a fluid- saturated porous solid. II. Higher frequency range. The Journal of the Acoustical Society of America (1956) 28:179-191.

[61] Schanz M. Wave propagation in viscoelastic and poroelastic continua. Lecture notes in applied mechanics, Springer, Vol. 2, (2001).

[62] Majchrzak E. and Turchan L. The general boundary element method for 3D dual-phase lag model of bioheat transfer. Engineering Analysis with Boundary Elements (2015) 50:76-82.

[63] Bonnet, G. and Auriault, J.L. Dynamics of saturated and deformable porous media, in N. Boccara and M. Daoud (eds.), Physics of Finely Divided Matter, Springer Proceedings in Physics, Springer, Berlin, Heidelberg (1985) 5:306-316.

[64] Bonnet G. Basic singular solutions for a poroelastic medium in the dynamic range. The Journal of the Acoustical Society of America (1987) 82:1758-1763.

[65] Messner, M. and Schanz M. A regularized collocation boundary element method for linear poroelasticity. Computational Mechanics (2011) 47:669-680.

[66] Majchrzak, E., and Turchan, L. Solution of dual phase lag equation by means of the boundary element method using discretization in time. Journal of Applied Mathematics and Computational Mechanics (2013) 12:89-96.

[67] Liao S. General boundary element method for non-linear heat transfer problems governed by hyperbolic heat conduction equation. Computational Mechanics (1997) 20:397-406.

[68] Liao S and Chwang A. General boundary element method for unsteady nonlinear heat transfer problems. Numerical Heat Transfer, Part B (1999) 35:225-242.

[69] Steinbach O. Numerical approximation methods for elliptic boundary value problems, Springer, (2008).

[70] Brenn G., Holzapfel G.A., Schanz, M. and Steinbach, O. Computation in engineering and science, Graz University of Technology, Vol. 5, (2009).

[71] Lubich C. Convolution quadrature and discretized operational calculus. I. Numerische Mathematik (1988) 52:129-145.

[72] Lubich C. Convolution quadrature and discretized operational calculus II. Numerische Mathematik (1988) 52:413-425. 
[73] Barra, L.P.S., Coutinho, A.L.G.A., Telles J.C.F. and Mansur W.J. Multi-level hierarchical preconditioners for boundary element systems. Engineering Analysis with Boundary Elements (1993) 12:103-109.

[74] Saad, Y. and Schultz, M.H. GMRES -- A generalized rain/real residual algorithm for solving nonsymmetric linear systems, SIAM J. Sci. Star. Computing (1986) 7:869-869.

[75] Freund, R.W. and Nachtigal, N.M. An implementation of the QMR method based on coupled two-term recurrences. SIAM Journal on Scientic Computing (1994) 15:313-337.

[76] Leonard J.B., Foster K.B. and Athey T.W. Thermal properties of tissue equivalent phantom materials. IEEE Transactions on Biomedical Engineering (1984) 31:533-536.

[77] Gilchrist, M.D., Murphy, J.G., Parnell, W. and Pierrat, B. Modelling the slight compressibility of anisotropic soft tissue. International Journal of Solids and Structures (2014) 51:3857-3865.

[78] Morrow, D.A., Donahue, T.L.H., Odegard, G.M. and Kaufman, K.R. Transversely isotropic tensile material properties of skeletal muscle tissue. Journal of the Mechanical Behavior of Biomedical Materials (2010) 3:124-129.

[79] Li. X., Li, C., Xue, Z. and Tian, X. Analytical study of transient thermo-mechanical responses of dual-layer skin tissue with variable thermal material properties. International Journal of Thermal Sciences (2018) 124:459-46. 